\title{
A NOTE ON THE VARIETY OF PLANE CURVES WITH NODES AND CUSPS
}

\author{
PYUNG-LYUN KANG
}

(Communicated by Louis J. Ratliff, Jr.)

\begin{abstract}
Let $\mathbf{P}^{N}$ be the projective space of all degree $d$ plane curves and $V(d, \delta, \kappa)$ the subvariety of $\mathbf{P}^{N}$ consisting of all reduced and irreducible curves with $\delta$ notes and $\kappa$ cusps. Then $V(d, \delta, \kappa)$ is irreducible if $\kappa \leq \frac{1}{2}(d+1)-g$.
\end{abstract}

Let $\mathbf{P}^{N}$ be the projective space parametrizing all plane curves of degree $d$ and $V(d, \delta, \kappa)$ the subvariety of $\mathrm{P}^{N}$ corresponding to all reduced and irreducible plane curves of degree $d$ with $\delta$ nodes and $\kappa$ ordinary cusps as their only singular points. Diaz and Harris [DH] showed that $V(d, \delta, \kappa)$ is a smooth variety of dimension $N-\delta-2 \kappa$ if $\kappa \leq 2 d-1$. In [K] we showed that $V(d, \delta, \kappa)$ is irreducible if $\kappa \leq 3$ except possibly when $\kappa=3$ and $d=5$ or 6 .

In this paper we prove

Theorem. The variety $V(d, \delta, \kappa)$ is irreducible if $\kappa \leq \frac{1}{2}(d+1)-g$.

This result goes along with the general suspicion that $V(d, \delta, \kappa)$ should be irreducible when $\kappa$ is pretty small with respect to $d$ while $\kappa$ approaches infinity as $d$ does. We remark here that there exist two components of $V(6,0,6)$ [Z].

We mean here by a node a point where a curve has two transverse smooth branches, and by a cusp a point where a curve has a singular branch, if it is locally given by $y^{2}=x^{3}$ we call it an ordinary cusp. The (geometric) genus of a curve is the genus of its normalization.

From now on we assume that $d$ is at least 5 since the irreducibility of all possible $V(d, \delta, \kappa)$ is known when $d<5$. We also work on the assumption that $\kappa \neq 0$; otherwise it is the Severi variety $V(d, \delta)$, the variety of reduced and irreducible plane curves of degree $d$ with $\delta$ nodes as their only singularities, which is irreducible $[\mathrm{H}][\mathrm{Z}]$.

Note that a plane curve of degree $d$ and geometric genus $g$ can be realized as a degree $d$ map $\varphi: C \rightarrow \mathbf{P}^{2}$, or equivalently, as a 3-dimensional subspace (a

Received by the editors May 31, 1988 .

1980 Mathematics Subject Classification (1985 Revision). Primary 14H10.

Key words and phrases. Plane curves, cusps, families of plane curves with nodes and cusps. 
$g_{d}^{2}$, for short) of $H^{0}(\mathscr{L})$ for a line bundle $\mathscr{L}$ of degree $d$ on a smooth genus $g$ curve $C$. From this point of view we consider $G_{d, \kappa}^{2}(C)$ the set of all $g_{d}^{2}$ 's on a smooth curve $C$ the associated maps $\varphi: C \rightarrow \mathbf{P}^{2}$ of which give rise to plane curves of degree $d$ with $\kappa$ cusps. Let $\mathscr{M}_{g}$ be the moduli space of genus $g$ curves and $G$ parametrize pairs $(C, V)$, where $C \in \mathscr{M}_{g}$ and $V \in G_{d, \kappa}^{2}(C)$. Then the theorem follows once we show that $G$ is irreducible and its general members give rise to plane curves in $V(d, \delta, \kappa)$. Since $\mathscr{M}_{g}$ is irreducible, it comes from the following lemma and its proof.

Lemma. Let $C$ be a smocth curve of genus $g$. Then $G_{d, k}^{2}(C)$ is irreducible of dimension $3 d-2 g-6-\kappa$ if $d \geq 2 g-1+2 \kappa$. If $V \in G_{d, \kappa}^{2}(C)$ is general and $\varphi_{V}: C \rightarrow \mathbf{P}^{2}$ is the associated map, then $\varphi_{V}(C)$, as a plane curve, has $\kappa$ ordinary cusps and nodes for the remaining singular points.

Proof. Let $G_{d, \kappa}^{2}(C, \mathscr{L})$ be all the $g_{d}^{2}$ 's associated to a fixed line bundle $\mathscr{L}$ of degree $d, d \geq 2 g-1+2 \kappa$, on $C$. We first show that it is irreducible.

Let $\varphi_{\mathscr{L}}=\varphi: C \rightarrow \mathbf{P}^{d-g}=\mathbf{P}\left(H^{0}(\mathscr{L})\right)$ be an embedding associated to $\mathscr{L}$. We may regard a linear series $V$ of dimension 2 (i.e., $\varphi_{V}: C \rightarrow \mathbb{P}^{2}$ ) associated to $\mathscr{L}$ as giving a rational map from $C$ to $\mathrm{P}(V)$, which is the composition of $\varphi$ and projection from the center $\mathbf{P}(V)^{\perp} \simeq \mathbf{P}^{d-g-3}$. Then by identifying $C$ with $\varphi(C), G_{d, \kappa}^{2}(C, \mathscr{L})$ can be considered as the set of all $(d-g-3)$-planes meeting $\kappa$ tangent lines of $C$ transversely away from $C$.

Let $C_{\kappa}$ be the $\kappa$ th symmetric product of $C$ and $\Delta$ the diagonal of $C_{\kappa}$, i.e., $\Delta=\left\{\left(p_{1}, \ldots, p_{\kappa}\right) \in C_{\kappa} \mid p_{i}=p_{j}\right.$ for some distinct $i$ and $\left.j\right\}$. Then $G_{d, \kappa}^{2}(C, \mathscr{L})$ becomes a fiber space with a fiber $F\left(p_{1}, \ldots, p_{\kappa}\right)$, over a point $\left(p_{1}, \ldots, p_{\kappa}\right) \in$ $C_{\kappa} \backslash \Delta$, the set of all $(d-g-3)$-planes meeting the tangent lines $t_{i}$ of $C$ at $p_{i}$ away from $C$.

Let $W_{3}\left(x_{i}\right)$ be all the $(d-g-3)$-planes containing a point $x_{i} \in t_{i}$, which is codimension 3 in $\operatorname{Gr}(d-g-3, d-g)$, the Grassmannian space of all $(d-g-3)$ planes in $\mathbf{P}^{d-g}$. Then

$$
F\left(p_{1}, \ldots, p_{\kappa}\right)=\bigcup_{\left\{\left(x_{1}, \ldots, x_{\kappa}\right) \mid x_{i} \in t_{i} \backslash\left\{p_{i}\right\}\right\}} \bigcap_{i=1}^{\kappa} W_{3}\left(x_{i}\right) .
$$

We now claim that $\bigcap_{i=1}^{\kappa} W_{3}\left(x_{i}\right)$ is irreducible of dimension $3(d-g-2-\kappa)$ and its closure is isomorphic to $\operatorname{Gr}(d-g-3-\kappa, d-g-\kappa)$ regardless of the choice of $p_{i}$ and $x_{i}$. Then $F\left(p_{1}, \ldots, p_{\kappa}\right)$ has a natural fiber space structure over $\kappa$ products of affine lines with an irreducible fiber $\bigcap_{i=1}^{\kappa} W_{3}\left(x_{i}\right)$. Therefore $F\left(p_{1}, \ldots, p_{\kappa}\right)$ is irreducible, so is $G_{d, \kappa}(C, \mathscr{L})$.

It now remains to prove the above claim. Note that the closure of $\bigcap_{i=1}^{\kappa} W_{3}\left(x_{i}\right)$ is the variety of all $(d-g-3)$-planes containing the plane that is spanned by $\left\{x_{1}, \ldots, x_{\kappa}\right\}$. Observe that any $\kappa$ tangent or secant lines are independent, i.e., they span $(2 \kappa-1)$-plane; if not some $\kappa$ tangent or secant lines would span 
at most $(2 \kappa-2)$-plane meeting $C$ at $2 \kappa$ points counting multiplicities, so the dimension of the hyperplanes containing these $2 \kappa$ points would be $d-g-$ $2 \kappa-1$. On the other hand $h^{0}\left(\mathscr{L} \otimes \mathscr{O}_{C}(-2 \kappa[p t]).\right)=d-2 \kappa-g+1$, which implies that the dimension of the hyperplanes containing $2 \kappa+2$ points is $d-2 \kappa-g$. Then this observation tells that $\left\{x_{1}, \ldots, x_{\kappa}\right\}$ spans $(\kappa-1)$-plane $S_{\kappa}$; otherwise $\left\{x_{1}, \ldots, x_{\kappa}, p_{1}, \ldots, p_{\kappa}\right\}$ would span at most $(2 \kappa-2)$-plane meeting $C$ at $2 \kappa$ points counting multiplicities. Then the set of all $(d-g-3)$-planes in $P^{d-g}$ can be identified with $\operatorname{Gr}\left(d-g-\kappa-3, \mathrm{P}^{d-g-\kappa}\right)$ which is irreducible of dimension $3(d-g-2-\kappa):$ consider the projection of $\mathbb{P}^{d-g}$ from the center $S_{\kappa}$ (cf. [K] Theorem (2.1)).

The irreducibility of $G_{d, \kappa}^{2}(C)$ follows from the facts, first, that $\operatorname{Pic}^{d}(C) \simeq$ $\operatorname{Jac}(C)$ is irreducible, second, that each fiber $G_{d, k}^{2}(C, \mathscr{L})$ over $\mathscr{L} \in \operatorname{Pic}^{d}(C)$ is irreducible.

For the last part of the lemma, note that, if $F_{i}=F_{i}^{0} \subset F_{i}^{1} \subset \cdots \subset F_{i}^{d-g}$ is the flag of the osculating planes of $C$ at $p_{i}$ and $\pi_{\Lambda}(C)$ has an ordinary cusp at $\pi_{\Lambda}\left(p_{i}\right)$, then $\Lambda$ satisfies $\operatorname{dim}\left(\Lambda \cap F_{i}^{3+j}\right)=j, j \geq 1$, and $\operatorname{dim}\left(\Lambda \cap F_{i}^{j}\right)=0, j \leq 3$ (cf. [EH] and [GH]). Such $\Lambda$ 's then clearly form a general locus of $\bigcap_{i=1}^{\kappa} W_{3}\left(x_{i}\right)$. For the nodes part, look at the projection $\pi_{\kappa}: \mathbf{P}^{d-g} \rightarrow \mathbf{P}^{d-g-\kappa}$ from $S_{\kappa}$ and the chodal variety $\mathscr{C}_{\kappa}(C)$ of $\pi_{\kappa}(C)$. Bertini then tells that a general $(d-g-\kappa-3)$ plane in $\mathbb{P}^{d-g-\kappa}$ must meet $\mathscr{C}_{\kappa}(C)$ at the right number of smooth points of $\mathscr{C}_{\kappa}(C)$ (and the locus of smooth points of $\mathscr{C}_{\kappa}(C)$ is contained in the union of the secant lines of $\left.\pi_{\kappa}(C)\right)$. Q.E.D.

Remark. In fact, the last part of the lemma follows also from the theorem of Diaz and Harris which is mentioned in the beginning of this paper (see [DH] for details).

Harris pointed out that there was another obvious case where $V(d, \delta, \kappa)$ is irreducible. Consider $V(d, \delta, \kappa)$. Since fixing each cusp and its tangential direction gives 5 linear conditions on the coefficients of plane curves of degree $d, V(d, \delta, \kappa)$ can be considered as a fiber space over $\mathbf{P}^{2 \kappa} \times \mathbf{P}^{\kappa}$ with a linear space fiber for big $d$.

\section{ACKNOWLEDGMENT}

I would like to thank Steven Diaz for leading me to this problem and this paper.

\section{REFERENCES}

[DH] S. Diaz and J. Harris, Ideals associated to deformations of singular plane curves, Trans. Amer. Math. Soc. (to appear).

[EH] D. Eisenbud and J. Harris, Divisors on general curves and cuspidal rational curves, Invent. Math. 74 (1983), 371-418.

[GH] P. Griffiths and J. Harris, Principles of algebraic geometry, Wiley-Interscience, New York, 1978. 
[H] J. Harris, On the Severi problem, Invent Math. 84 (1986), 445-461.

$[\mathrm{K}] \mathrm{P}$. Kang, On the variety of plane curves of degree $d$ with $\delta$ nodes and $\kappa$ cusps, Trans. Amer. Math. Soc. (to appear).

[R] Z. Ran, On nodal plane curves, Invent. Math. 86 (1986), 529-534.

[Z] O. Zariski, Algebraic surfaces, Springer-Verlag, Berlin, 1971.

Department of Mathematics, Pohang Institute of Science and Technology, Pohang, KOREA 